\title{
Videoconferencing Design for Remote Groups
}

\author{
Petr Slovák \\ Lab. of Advanced Network Technologies \\ Faculty of Informatics \\ Masaryk University \\ Botanická 68a, 60200 Brno \\ Czech Republic \\ E-mail: slovak@ics.muni.cz
}

\author{
Eva Hladká \\ CESNET z. s. p.o. \\ Zikova 4, 16000 Prague \\ Lab. of Advanced Network Technologies \\ Faculty of Informatics \\ Masaryk University \\ Botanická 68a, 60200 Brno \\ Czech Republic \\ E-mail: eva@fi.muni.cz
}

\author{
Pavel Troubil \\ Lab. of Advanced Network Technologies \\ Faculty of Informatics \\ Masaryk University \\ Botanická 68a, 60200 Brno \\ Czech Republic \\ E-mail: pavel@ics.muni.cz
}

\begin{abstract}
Videoconferencing has emerged as a popular method of remote communication. The design of a videoconferencing environment strongly influences the communication efficiency and user satisfaction. Various studies on perceived effects of videoconferencing environments and their parameters have been done, however, they primarily focused on dyadic conversation. This paper describes an exploratory study on the effects on remote groups communication. Beginning with the standard environment, a videoconferencing design has been developed iteratively according to information obtained from the experimental group. Two new videoconferencing environment designs are introduced to suit the group conversation needs better and bring the group videoconference closer to face-to-face communication.
\end{abstract}

\section{INTRODUCTION}

Videoconferencing is an increasingly popular way of coworking independently of physical distance. Although there has been a remarkable success in enhancing the quality of audio and video components of videoconferencing environments, it has yet to achieve the standards of face-to-face communication in overall efficiency and subjective impression of the participants.

While the principles of dyadic conversation have been pursued to great depths, nearly no research has focused on another very common videoconferencing layout - conversation between two or more remote groups ${ }^{1}$. The usual videoconferencing design, therefore, treats videconferences with remote groups similarly to those with single participants - the whole group is usually shot by one camera while videostreams (and eventually other data) from remote locations are projected on to one shared screen ${ }^{2}$. With this approach, the problems found in dyadic communication (such as loss of eye contact and other nonverbal cues) can become even more salient.

In this paper, we propose new designs for communication of remote groups with special care given to achieving a short learning curve, improving user satisfaction and usability while minimising hardware and room requirements.

\footnotetext{
${ }^{1} \mathrm{~A}$ remote group is understood in this paper as a group of 3 or more people located in one room that are, to some extent, sharing common videoconferencing equipment (e.g., screen, sound system etc.).

${ }^{2}$ Throughout the paper, we will reference this type of videoconferencing environment as the standard design
}

The structure of the paper is as follows: the next section gives a summary of work closely related to this topic. Section 3 then states our goals and Section 4 will introduce videoconferencing tools used in our designs. The following two sections explain the chosen methodology and obtained results, respectively. Finally, Section 7 introduces open problems for future work and the last section concludes the paper.

\section{RELATED WORK}

Numerous studies have focused on determining how different aspects of videoconferencing environments affect task results and the feelings of the participants, e.g., [1], [2], [3], [4], [5], [6], [7], [8]. Some widely accepted results are:

- Keeping reasonable framerate (above $16 \mathrm{fps}$ ) is very important [6], [7].

- Changing the size and/or resolution of the video window did not make any measurable difference either in task results or subjective view of the participants [1], [7], [8].

- Desynchronisation of audio and video stream (greater than $80-100 \mathrm{~ms}$ ) is lethal to fluent conversation [8].

- Sound quality seems to be much more important than video stream quality. The videoconferencing advantages are more significant for larger groups [1], [2], [5], [7].

- The videostreams should include the whole torso of the taken participant rather than just the head.

Most of the above mentioned studies (with the exception of [2], where the conversation was between two couples) have used only dyadic designs. Although there are some papers introducing/describing videoconferencing systems for communication of remote groups, e.g., [9], they are based on personal experience of the researchers. No purely experimental work on the topic of remote group videoconferencing is known to the authors.

To support decision making in groups, either co-located or remote, the Group Support Systems (GSS) have been developed [10], [11]. GSS is a software environment consisting of several tools facilitating, e.g., idea generation, idea categorisation, and voting. The videoconferencing environments may be utilised together with a Group Support System. 


\section{GOALS}

Our goal is to design a group-oriented videoconferencing environment that would fulfil the following properties:

- To keep the learning curve reasonably short, users should be able to use the environment without any knowledge or experience with similar systems. The perceived differences to face-to-face communication should be kept to minimum.

- To support the communication style needed for solving "quality" problems. No verifiable right solution exists in these problems and all sides have to agree on a reasonable compromise. Such problems are very frequent in both business and academic discussions.

- To keep the videoconferencing environment as versatile as possible, we have decided to focus mainly on supporting communication and not on any additional background materials support ${ }^{3}$ (as these are strongly dependent on type of the task) - therefore, the camera placement and arrangement of the viewing screen were of main interest.

- To ease deployment, the final setup should not be too expensive or immobile - it should take at most few hours to set up at a new location.

\section{VIDEOCONFERENCING TOOLS AND ENVIRONMENTS}

For this study, we needed videoconferencing tools and an environment that would allow us to make changes very quickly and effortlessly depending on evolution of the study without large demands on software and hardware development.

This problem can be divided into two parts: the network environment layer and videoconferencing client tools layer.

\section{A. Network environment layer.}

The network environment layer takes care of interconnecting all the participants of the videoconferencing environment into a continuous network. There are two possible approaches to solve this: (1) using multicast connectivity or (2) userempowered software based data replication using reflectors.

Although the multicast solution scales very well, it has several disadvantages, including low coverage of multicast and our inability to influence this part of the environment, e.g., to optimise data stream distribution with respect to a communication schema. Because of these reasons we have opted for the second solution - the application-level distribution unit called a reflector. A similar concept is used in many systems such as H.323 MCUs $^{4}$. The modular userempowered UDP packet reflector used in this study has been developed in our laboratory [12]. It is highly configurable with modules loadable in run-time, supporting sophisticated access control policing and even data transcoding for some data formats. Media streams may be encrypted by the client software tools using symmetric encryption in the event that the data replication site is not considered trusted. In order to mitigate the problem with capacity and inherent unreliability

\footnotetext{
${ }^{3}$ With an exception for a short shared workplace experiment.

${ }^{4}$ Multipoint Control Units
}

of a central unit, it is possible to decentralise a reflector into a network of reflectors [13].

\section{B. Client tools layer}

The client layer comprises of tools and hardware devices on client side. The software tools primarily incorporate audio, video, and a shared workplace. For the videoconferencing clients we had to choose a client compatible with our UDP packet reflector and flexible enough to allow us to implement changes during the study.

Basic videoconferencing capabilities were provided by MBone Tools ${ }^{5}$. Robust Audio Tool (RAT) was used for audio transmission and playback. RAT supports a variety of audio codecs and allows the user to fine tune the audio stream based on quality requirements or bandwidth limitations as appropriate. Video communication was provided by Videoconferencing Tool (VIC) enabling transmissions of video acquired from video capture cards or USB cameras. The both tools allow for a wide range of transmission quality and bandwidth requirements settings.

\section{Methodology}

To fulfil the goals set above, it is vital to know which parts of the videoconferencing environment are important to the users. With no relevant research on this topic, exploratory, user-centred research design seemed the best choice [14]. Our study is based on longitudinal (5 month) cooperation with a group of students where the focus group technique was used as the main method of obtaining information.

A focus group is a common method of data collection in qualitative research, in which a group of 6-8 people are interviewed by a skilled moderator about their attitude towards an issue, product, or service. The participants are free to talk with other group members, as the group interaction is the primary data source. More detailed information can be found in [15], [16].

We have worked with a group of 10 students attending a digital media course during the spring semester with meetings held every two to three weeks (with few exceptions due to holidays, etc.) The basic idea was to iteratively develop videoconferencing environments according to suggestions from the users.

On each meeting the students first used the prepared videoconferencing environment to discuss some problems related to their digital media course. This lasted usually slightly more than one hour. Then, a hour long focus group took place.

During these focus groups, students exchanged their impressions of the environment and then, as a group, tried to express their view of the problematic parts of the proposed environment. Afterwards, the whole group looked for possible solutions. If the proposed environment modifications were feasible, we incorporated them into the videoconferencing environment used in the next meeting.

\footnotetext{
${ }^{5}$ http://www-mice.cs.ucl.ac.uk/multimedia/software/
} 


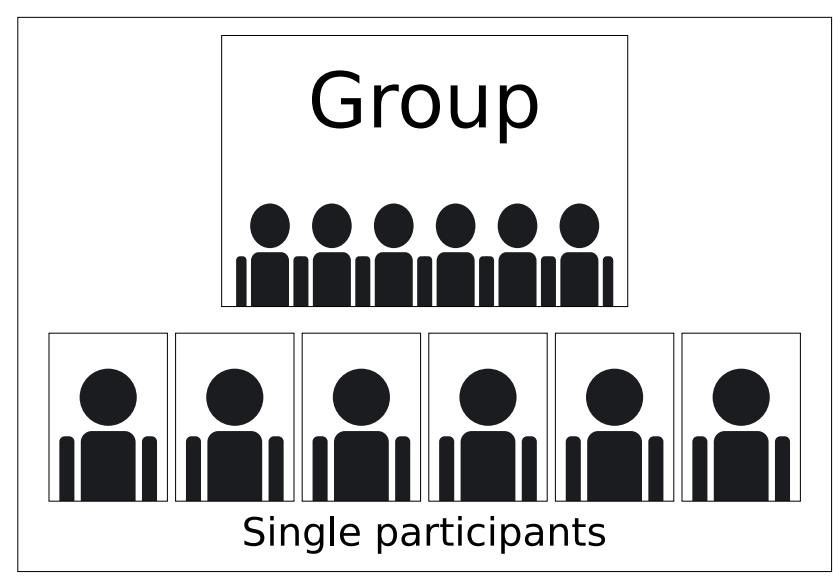

Figure 1. Diagram of the first design

For example, the topics for the first meeting were as follows ${ }^{6}$ :

- Perception of the feedback from other participants.

- Fluency of the conversation compared to face-to-face.

- Effect of the environment on required concentration and understanding the discussion (if high concentration is needed even a short discussion can be very tiring).

- Perceived difficulty to focus directly on a certain participant.

- Evaluation of the proposed screen arrangement.

\section{REsults}

The proposed two group oriented videoconferencing environments differ in the extent to which the users can individually alter the arrangement of videostreams from the remote (or co-located) group. The first design has been already implemented and tested, the second is still in the pre-implementation phase.

As was shortly stated in the Goals section, our work focuses primarily on providing the users with the possibility to see and hear each other in the best possible way. We therefore assume, that sharing of the underlying materials and other group support services (such as collaborative word processors, voting systems or slide synchronisation etc.) are supported by specialised software packages.

\section{A. First design}

1) Intuition: The basic idea of this design is to allow users to concentrate on individual members of the remote group while still keeping one shared screen for the whole group. As can be seen on diagram (Fig. 1), the standard image of the whole remote group is kept (in the focus groups meetings, participants repeatedly expressed that this "whole room view" is very important for them), while video streams displaying individual remote participants are added.

\footnotetext{
${ }^{6}$ The other meetings were similar in style and closely connected to the current changes in design. For detailed information on precise topics please contact the authors.
}

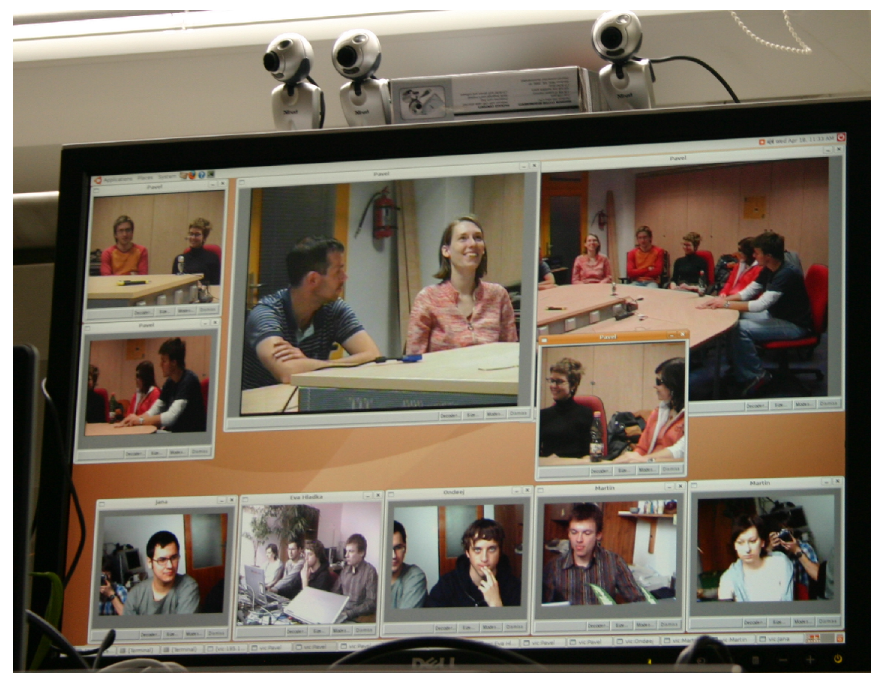

Figure 2. Implementation of the first design

2) Implementation: One of the groups was placed in a videoconferencing room satisfying the AccessGrid Point requirements ${ }^{7}$. The whole group view was recorded by a tracking camera and SHURE Easyflex EZB/C microphones provided the audio acquisition. A LCD projector was used for the visualisation of video outputs.

The other group was placed in a small conference room using a mobile videoconferencing setup. This setup consists of a computer equipped with a USB camera for the whole group view and a ClearOne AccuMic PC echo-cancelling microphone. Video outputs were visualised by a 24" HD resolution LCD monitor. In both rooms, standard, low-priced web cameras recorded the individual participants.

In some cases, one or two individual users were connected to the videoconferencing environment by a laptop, USB camera and headset.

For a shared workplace experiment, we equipped one of the groups with Sympodium ID350 interactive pen display and Canopus TwinPact 100 converter, allowing the users to utilise the shared workplace via standard video stream and VIC.

All of the video streams were processed by the VIC software and sent to the reflector. Fig. 2 shows the implementation in action.

On personal computers only VIC software and camera drivers have to be installed - this usually takes no more than 10 minutes and has to be done only once. Assuming that all of the participants have their personal laptops already functional, the whole setup requires only one standard machine with a mid-level camera, good audio equipment ${ }^{8}$ and few possibly low-end web cameras.

As can be seen, this environment fulfils our fourth goal of mobility and low costs.

\footnotetext{
${ }^{7}$ http://www.accessgrid.org/hardware

${ }^{8}$ Since the videoconference sound quality is crucial for the users weariness and whole environment satisfaction, high quality microphones and echocancelling equipment is required for successful system application.
} 


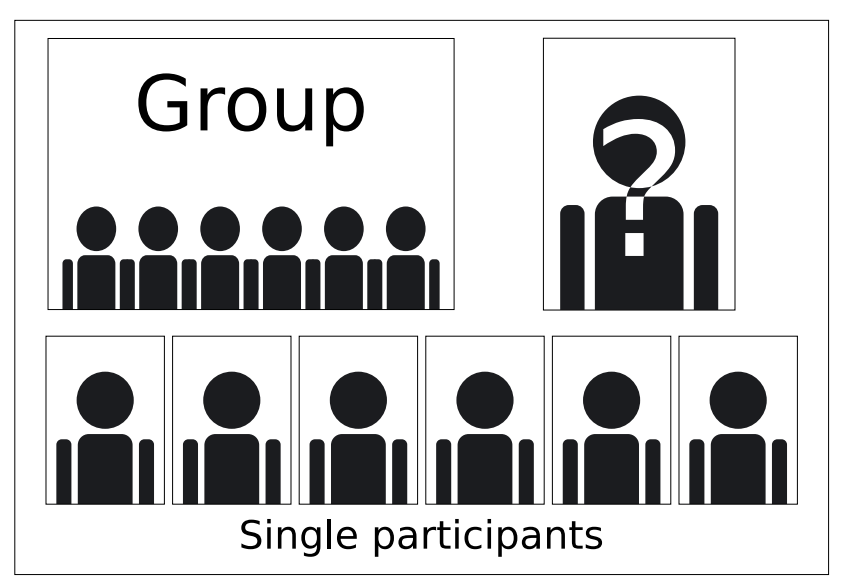

Figure 3. Diagram of participant's screen in the second design

3) Possible modifications: Some of the users in our testing group also wanted to include videostreams of members from the co-located group together, similar to the remote participants. This helped them to overcome the barrier between colocated/remote group and also helped to create a vision of one connected group.

Conversely, some users (even if they also experienced the above stated effect) felt so uneasy by seeing themselves on the screen that they decided not to show the co-located participants at all.

The positive/negative effect seems to vary between different groups - it is best to let the users try both options and then pick the more convenient one.

4) Evaluation: This environment was found to be superior to the standard design by the users of our groups. Participants found it easier to perceive feedback from other users, felt that less concentration was needed to follow the conversation and the discussion was more fluent.

\section{B. Second design}

1) Intuition: Contrary to the face-to-face conversation, the users are not able to view selected participants in more detail independently of the current focus of others ${ }^{9}$. This lack of selective focus was perceived by the students as one of the major drawbacks of videoconferencing environments. This design tries to provide the users with more control over the presented video streams and therefore, hopefully, diminish the perceived problem.

Rather than projecting the videostreams on one shared screen, each participant uses his or her laptop as the visualisation device. A shared screen can be then used for slides or any other material that the group needs. The same way as in the previous design, one camera captures the image of

\footnotetext{
${ }^{9}$ In some videoconferencing designs, it is possible to remotely control the camera and therefore focus on one or more selected participants (e.g., current speaker). Unfortunately, this restricts the view of all the users to these selected participants even if some would prefer to concentrate their attention on other group member or members. Possible solution of multiple remotely controlled cameras is too demanding technically.
}

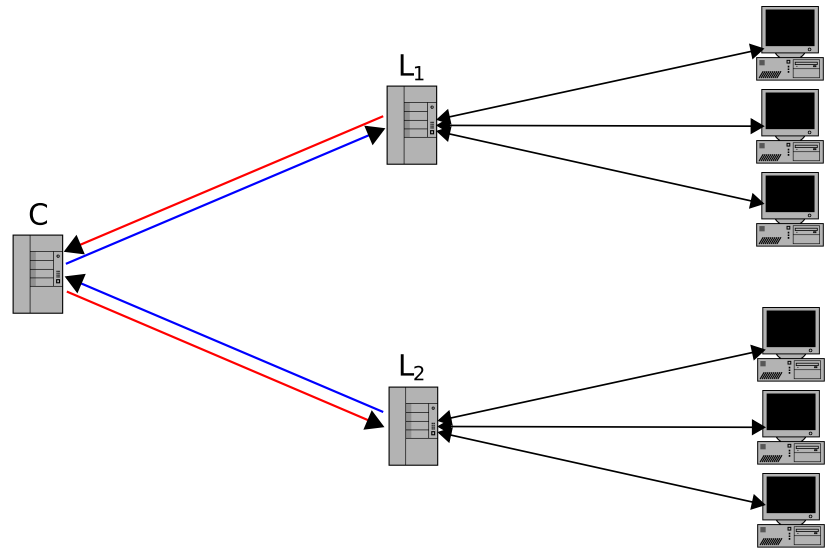

Figure 4. Diagram of reflector network for two remote groups in the second design

the whole group and individual participants are recorded by smaller cameras.

The basic structure diagram of the visualisation screen available to each user is shown at Fig. 3. With a hotkey assigned to each of the small videostream windows on the bottom (or simply by clicking on them), user can quickly select the individual participant (either remote or co-located) that should be shown in more detail in the top right part of the screen.

This freedom of selective focus could, according to the students from our test group, make the videoconferencing environment similar to face-to-face collaboration - and therefore also more satisfying and pleasant for the users.

2) Future implementation: One possible implementation is based on the use of network of reflectors as can be seen on Fig. 4. The advantage of this implementation is optimising bandwidth usage, as it is more efficient for the streams of colocated participants to be sent over the Local Area Network.

The basic idea is to provide each group with a local reflector in order to distribute streams of co-located participants through the local network. Besides the distribution to local participants, all streams from local reflectors (denoted by $L_{1}, L_{2}$ ) are forwarded to a central reflector $C$. The bandwidth saving is then implemented by configuring the central reflector not to send back any stream to a local reflector it was received from. This ensures that local groups do not receive their own streams once again.

Furthermore, this solution provides the capability for effortless videoconferencing of more than two remote groups. Additional groups may simply be added by connecting additional local reflectors.

Since current VIC implementations do not support the selection of a user that should be displayed in more detail, development of a plugin for VIC is needed to allow for this functionality on personal computers. 


\section{FUTURE WORK}

Our current work is aimed at a verification of the general hypotheses obtained from the test group. We would like to have the assumptions confirmed by employing several testing groups attending less (2-3) focus groups meetings than our experimental group did. The information gained through these focus groups may lead to some minor changes in the proposed designs.

Implementation of the second design will occur simultaneously with the hypotheses verification. After the development of the second design is finished, a formal quantitative testing of the environments will take place. We plan to compare the environments in terms of communication effectiveness and member satisfaction.

In the end of the first research period we prepared two modifications of standard videoconferencing environment. The first one employing stereoscopic video and the second one using HD resolution video with a "common table" room design ${ }^{10}$. These systems have been prepared for future testing.

One possible direction for future software development is the creation of a semi-automatic tool for optimal videostream window placement, allowing the user to adjust window placement according to his or her own preferences.

For the more remote future we considered the integration of a videoconferencing environment into a Group Support System and developing other parts of GSS.

\section{CONCLUSIONS}

We have qualitatively tested the standard videoconferencing environment using an experimental group. On the basis of information obtained, we proposed two new group oriented videoconferencing designs.

The first one was successfully implemented, while the second one is in the developmental phase. Both the environments were found superior to the standard environment by all members of our test group, but it is hard to generalise to larger populations due to the experiment's exploratory design.

Formal quantitative testing should now take place to compare the standard and new designs with each other.

As a secondary effect, the members of our experimental group with no previous videoconferencing experiences started to use the environment on their own without any special training.

\section{ACKNOWLEDGEMENTS}

This project has been supported by a research intent "Optical Network of National Research and Its New Applications" (MŠM 6383917201) and "Parallel and Distributed Systems"

\footnotetext{
${ }^{10}$ In this design, camera and projection screen are placed very close to the group's table. The remote group's table may be then perceived as the opposite side of a long negotiation table.
}

(MŠM 0021622419). The authors would like to acknowledge help of Pavel Šiler for his assistance with camera systems and video records processing of the focus groups.

\section{REFERENCES}

[1] A. H. Anderson, A. Newlands, J. Mullin, A. Fleming, G. DohertySneddon, and J. M. van der Velden, "Impact of video-mediated communication on simulated service encounters." Interacting with Computers, vol. 8, no. 2, pp. 193-206, 1996.

[2] O. Daly-Jones, A. Monk, and L. Watts, "Some advantages of video conferencing over high-quality audio conferencing: Fluency and awareness of attentional focus," International Journal of Human-Computer Studies, vol. 49, no. 1, pp. 21-58, 1998.

[3] C. O'Malley, S. Langton, A. H. Anderson, G. Doherty-Sneddon, and V. Bruce, "Comparison of face-to-face and video-mediated interaction," Interacting with Computers, vol. 8, no. 2, pp. 177-192, 1996.

[4] G. Doherty-Sneddon, A. H. Anderson, C. O’Malley, S. Langton, S. Garrod, and V. Bruce, "Face-to-face and video-mediated communication: A comparison of dialogue structure and task performance," Journal of Experimental Psychology: Applied, vol. 3, no. 2, pp. 105-125, 1997.

[5] A. Sanford, A. H. Anderson, and J. Mullin, "Audio channel constraints in video-mediated communication." Interacting with Computers, vol. 16, no. 6, pp. 1069-1094, 2004.

[6] M. Vitkovitch and P. Barber, "Effect of video frame rate on subjects' ability to shadow one of two competing verbal passages," Journal of Speech, Language, and Hearing Research, vol. 37, no. 5, pp. 12041210, 1994.

[7] A. H. Anderson, L. Smallwood, R. MacDonald, J. Mullin, and A. Fleming, "Video data and video links in mediated communication: what do users value?" International Journal of Human-Computer Studies, vol. 52, no. 1, pp. 165-187, 2000.

[8] V. Bruce, "The role of the face in communication: Implications for videophone design." Interacting with Computers, vol. 8, no. 2, pp. 166 176, 1996.

[9] A. Girgensohn, J. Boreczky, P. Chiu, J. Foote, L. Wilcox, and S. Smoliar, "Supporting group-to-group collaboration in videoconferences," in HICSS '02: Proceedings of the 35th Annual Hawaii International Conference on System Sciences (HICSS'O2)-Volume 4. IEEE Computer Society, 2002, p. 112.

[10] J. F. Nunamaker, A. R. Dennis, J. S. Valacich, D. R. Vogel, and J. F. George, "Electronic meeting systems to support group work." Communications of the ACM, vol. 34, no. 7, pp. 40-61, 1991.

[11] J. Jay F. Nunamaker, R. O. Briggs, D. D. Mittleman, D. R. Vogel, and P. A. Balthazard, "Lessons from a dozen years of group support systems research: a discussion of lab and field findings," Journal of Management Information Systems, vol. 13, no. 3, pp. 163-207, 1996.

[12] E. Hladká, P. Holub, and J. Denemark, "An active network architecture: Distributed computer or transport medium," in 3rd International Conference on Networking (ICN'04), Gosier, Guadeloupe, Mar. 2004, pp. 338-343.

[13] P. Holub, E. Hladká, and L. Matyska, "Scalability and robustness of virtual multicast for synchronous multimedia distribution," in Networking - ICN 2005: 4th International Conference on Networking, Reunion Island, France, April 17-21, 2005, Proceedings, Part II, ser Lecture Notes in Computer Science, vol. 3421/2005. La Réunion, France: Springer-Verlag Heidelberg, Apr. 2005, pp. 876-883. [Online]. Available: http://www.springerlink.com/index/GETV62MG4GA0CUPL

[14] E. R. Babbie, The Practice of Social Research, 9th ed. Stamford, CT: Wadsworth, 2001

[15] R. A. Krueger and M. A. Casey, Focus Groups: A Practical Guide for Applied Research. Sage Publications, 2000.

[16] D. L. Morgan, "Focus groups," Annual Review of Sociology, vol. 22, pp. 129-152, 1996. 